\title{
Correction to: Spatiotemporal Distribution of Radionuclides in Soil in the Tokyo Metropolitan Area
}

\section{Correction to:}

\section{H. Yamazaki, Radioactive Contamination of the Tokyo}

Metropolitan Area, https://doi.org/10.1007/978-981-15-7368-2_3

The book was inadvertently published with errors in Table 3.4 in Chap. 3. It appears correctly in the eBook version, and only the print version has been updated. 